\title{
A Hybrid Data Dependent Dissimilarity Measure for Image Retrieval
}

\author{
Hamid Shojanazeri \\ Faculty of Science and Technology \\ School of Engineering and Information Technology \\ Federation University Australia, Gippsland, Australia \\ hshojanazeri@federation.edu.au
}

\author{
Shyh Wei Teng, Guojun Lu \\ Faculty of Science and Technology \\ School of Engineering and Information Technology \\ Federation University Australia, Gippsland, Australia
}

\begin{abstract}
In image retrieval, an effective dissimilarity measure is required to retrieve the perceptually similar images. Minkowski-type $\left(l_{p}\right)$ distance is widely used for image retrieval, however it has its limitations. It focuses on distance between image features and ignores the data distribution of the image features, which can play an important role in measuring perceptual similarity of images. $l_{p}$ also favours the most dominant components in calculating the total dissimilarity.

A data dependent measure, named $m_{p}$-dissimilarity, which estimates the dissimilarity using the data distribution, has been proposed recently. Rather than relying on geometric distance, it measures the dissimilarity between two instances in each dimension as a probability mass in a region that encloses the two instances. It considers two instances in a sparse region to be more similar than in a dense region. Using the probability of data mass enables all the dimensions of feature vectors to contribute in the final estimate of dissimilarity, so it does not just heavily bias towards the most dominant components. However, relying only on data distribution and completely ignoring the geometric distance raise another limitation. This can result in finding two instances similar only due to being in a sparse region, however if the geometric distance between them is large then they are not perceptually similar. To address this limitation we proposed a new hybrid data dependent dissimilarity (HDDD) measure that considers both data distribution as well as geometric distance. Our experimental results using Corel database and Caltech 101 show that $(H D D D)$ leads to higher image retrieval performance than $l_{p}$ distance $\left(l_{p} D\right)$ and $m_{p}$.
\end{abstract}

Keywords-Image retrieval; Dissimilarity measure; Data dependent dissimilarity measure

\section{INTRODUCTION}

With development of the Internet and the advancement of image capturing devices along with the cheaper memory devices, the size of digital image collection is increasing rapidly. So, efficient and robust image retrieval system is increasingly in demand by different domains, e.g. fashion, crime prevention, publishing, medicine, and architecture, in order to make use of the images in a large database effectively. In image retrieval, an image is presented to a database as a query and a set of perceptually similar images are retrieved. An effective image retrieval system requires images to be represented by a robust and discriminative feature vector/descriptor. Images can be described using different low-level features such as colour, shape and texture $[1,2]$. In addition, an effective dissimilarity measure plays an important role in comparison of the query image feature vector and those of the stored images. Most researchers employ the Minkowski-type $\left(l_{p}\right.$-norm $)$ metric, particularly the $\left(l_{2}\right.$-norm): well known as Euclidean Distance (ED), as the dissimilarity measure [3].

One of the challenging problems in image retrieval is the selection of an effective dissimilarity measure to compare the images. $l_{p} D$ is the main dissimilarity measure in many applications, such as data mining, clustering, and image retrieval [4]. As we will explain in greater detail in Section III, $l_{p} D$. has two main limitations: (1) it focuses on distance between features of images and ignores the data distribution of image features, which can play an important role in measuring perceptual similarity of images; and (2) $l_{p} D$ favours the most dominant components in calculating the total distance.

Psychologists have highlighted the important role of data distribution in humans perceiving similarity between instances in a dataset. They argued that the dissimilarity between two instances is influenced by other instances in the dataset. Two instances in a relatively dense area are perceptually less similar than two instances of the equal distance in less dense area [5]. For example two red apples among green apples perceptually look more similar than the same two red apples among other red apples. Based on this idea, a data dependent dissimilarity measure called " $m_{p}$-dissimilarity" has been proposed to address the problems with $l_{p} D[3]$.

This data dependent dissimilarity measure [3] calculates the dissimilarity between two instances using data distribution in the dataset instead of geometric distance as used in $l_{p} D$. Basically in this method, the value calculated to indicate the dissimilarity between two instances will also take into account their dissimilarity to the other instances in the dataset. A region is defined between two instances. Two instances are less dissimilar if there are not many other similar instances falling in this region and they are more dissimilar if the number of similar instances is large.

The data dependent dissimilarity measure has shown promising results in classification of data such as music, text and digits. In this work we will investigate the performance of $m_{p}$-dissimilarity in image retrieval. To evaluate this method, we use two datasets, which are represented with two different 
sets of features, colour histograms and local binary patterns (LBP). We use colour histograms as they are more intuitive to explain $l_{p} D$ limitations and $m_{p}$ strengths on Corel dataset as is discussed in following sections. Also, we used Caltech 101, represented by LBP features as a real world dataset to evaluate $m_{p}$ performance.

The rest of the paper is organised as follows, two dissimilarity measures: $l_{p} D$ and $m_{p}$-dissimilarity, are discussed in Section II. The experimental study results are provided in Section III, followed by conclusions in the last section.

\section{DISSIMILARITY MEASURE BASED ON GEOMETRIC MODELS}

In image retrieval, images are represented using feature vectors. To retrieve a similar set of images, an effective dissimilarity measure must be used to compare the feature vectors. In the following, we will review an existing dissimilarity measure commonly used in image retrieval and a new dissimilarity measure.

A wide range of geometric dissimilarity measures are discussed in [4]. [6, 7] have each provided a comprehensive analysis and comparison of the dissimilarity metrics in image retrieval. The study in [6] has compared the performance of Histogram Intersection, Minkowski-form, Quadratic and Mahalanobis Distance. Its results have shown that ED has achieved the best retrieval results. [7] has compared the performance of sum of squared of absolute differences (SSAD), sum of absolute difference, maximum value, Canberra, city block, Minkowski $(p=3)$ and ED on the same version of Corel database [8] which has been used in this work. Its results have also shown that the ED is the most suitable dissimilarity metric for image retrieval.

Generally, the distance between two d -dimensional vectors $\mathrm{x}$ and $\mathrm{y}$ based on $l_{p} D$ is defined as follows [1]:

$$
l_{p}(x, y)=\|x-y\|_{p}=\left(\sum_{i=1}^{d} a b s\left(x_{i}-y_{i}\right)^{p}\right)^{1 / p}
$$

where $p>0,\|.\|_{p}$ is the $p$ order norm of a vector, $x_{i}$ and $y_{i}$ are the $i^{\text {th }}$ component of a vector and $a b s($.$) is the absolute$ value. The limit condition is defined as:

$$
l_{\infty}(x, y)=\|x-y\|_{\infty}=\max _{i} \operatorname{abs}\left(x_{i}-y_{i}\right)
$$

$l_{p} D$ is a popular choice of distance function as it intuitively corresponds to the distance defined in the real threedimensional world. It has been widely used in many image retrieval systems as the dissimilarity measure to compare the feature vectors derived from images [9-13].

However, $l_{p} D$ has its limitations, it measures the distance between image features of two images and completely ignores the distribution of other image features in the dataset. However, the distribution of image features considerably impacts the perceptual similarity between images as it is shown in the following example. Consider two red apples among the many green apples; the red apples perceptually are more similar to each other than green apples. However, considering another data distribution where the two red apples are among other red apples. In this distribution, all the red apples perceptually are similar to each other and the similarity of that two red apples is perceptually more difficult to be spotted. So the data distribution impacts the perpetual similarity of images.

The other limitation of $l_{p} D$ is that it favours the most dominant components in calculating the total dissimilarity. This characteristic of $l_{p} D$ results in negligible contribution of feature dimensions that have small values compared with dimensions that have dominant values [14]. This has negative impact when we are looking for the similar objects, e.g. objects in different backgrounds. Using the colour histograms in this work, the image retrieval results based on $l_{p} D$ will be heavily bias the towards the dominant colours (e.g. ones of the background), so the detailed colours within the objects in the images might not have the satisfactory level of influence in retrieving the closest match. Following the previous example, consider the two red apples among green apples, however this time one of red apples is located on a black background and rest of apples on white background. Although, the red apples are perceptually more similar, $l_{p} D$ finds the greatest distance between red apples in white (query) and black backgrounds. This is the result of the great difference between the black background, which is the dominant colour located in the first bin and the white colour located at the last bin of histogram.

\section{DATA DEPENDENT DissimiLARITY MEASURE}

To address the discussed limitations of $l_{p} D$, a data dependent dissimilarity measure have been proposed [3]. This measure is called $m_{p}$-dissimilarity and it focuses on the data distribution of the dataset instead of simply measuring the distance. It has been performed equal or better than $l_{p} D$ in context of information classification and retrieval problems. This method has been evaluated using text, music, digits and artificial datasets.

This idea is based on the distance-density model proposed by Krumhausl [5] which prescribes that two instances in a sparse region are more similar than two instances in a dense region. To measure the dissimilarity between two instances: $x$ and $y$, it defines a region between them and search for other instances in the dataset that falls in this region. So, the data distribution plays the main role to determine the number of instances similar to $\mathrm{x}$ and $\mathrm{y}$ that fall in this region. If this number is large, then $m_{p}$ considers it as a dense region, and therefore $\mathrm{x}$ and $y$ are less similar. Vice versa, if the number is small, then $\mathrm{x}$ and $\mathrm{y}$ are in sparse region and are considered more similar.

In order to measure the dissimilarity between $\mathrm{x}$ and $\mathrm{y}$, $m_{p}$ considers the relative positions of $\mathrm{x}$ and $\mathrm{y}$ with respect to the rest of the data distribution in each dimension. The dissimilarity between $\mathrm{x}$ and $\mathrm{y}$ in dimension $i$ can be estimated as the probability data in the region $R_{i}(x, y)$ that encloses $\mathrm{x}$ and $\mathrm{y}$. If there are many instances in $R_{i}(x, y)$, then it will be considered as a dense region. Therefore $\mathrm{x}$ and $\mathrm{y}$ are likely to be more dissimilar in dimension $i$. Using the same power 
mean formulation as in $l_{p}$ - norm, the data dependent dissimilarity measure based on probability mass can be defined as:

$$
m_{p}(x, y)=\left(\frac{1}{d} \sum_{i=1}^{d} a b s\left(\frac{\left|R_{i}(x, y)\right|}{N}\right)^{p}\right)^{1 / p}
$$

where $\left|R_{i}(x, y)\right|$ is the data mass in which is the number of instances that fall in the region of $R_{i}(x, y)$, and $n$ is the number of instances in the dataset. The enclosing region is defined as follows. $R_{i}(x, y)=\left[\min \left(x_{i}, y_{i}\right)-\sigma, \max \left(x_{i}, y_{i}\right)+\right.$ $\sigma$ ], and $\sigma$ is a small number $\sigma \geq 0$. Although $m_{p}$ employs the same power mean formulation as $l_{p}$, the core calculation is based on mass rather than distance. It signifies the degree of dissimilarity: the higher the measure, the more dissimilar the two instances are; just like $l_{p}$.

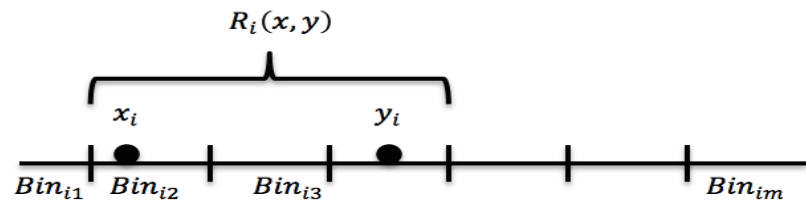

Fig.1. $\boldsymbol{R}_{\boldsymbol{i}}(\boldsymbol{x}, \boldsymbol{y})$ Defined Region between Two Instances using bins

Calculation of $m_{p}$ is expensive as it requires a range search in each dimension, so to address this problem a new implementation has been propose in [15]. In this new implementation a histogram is used, the real values in each dimension $i$ are divided into $m$ bins. The number of points in each bin is computed as a preprocessing step, and then data mass between two points can be computed using number of bins between them. An illustration of defining $R_{i}(x, y)$ using bins implementation is shown in Fig 1.

To calculate the data mass between the query and each of the stored images, in each dimension of the colour histograms (96 dimensions representing RGB channels), a region is defined using the values of query and each stored image. The neighbourhood of the region, as shown in Fig.1, is the standard deviation of all values in dataset of that dimension. The number of other images that their values in that dimension of colour histogram falling in the defined region is considered as data mass. As discussed before, the sparser data mass leads to a higher similarity.

As this method works based on the distribution of image feature vectors instead of only considering the distance between each dimension of them, it can address the $l_{p} D^{\prime} s$ limitation with being in favour of the most dominant components. $m_{p}$ also considers the dissimilarity of the two instances with the rest of the data in dataset. Generally in a data distribution which has many similar images but are different in their details and these details play a more important role as compared with the rest of image, $m_{p}$ is more effective in retrieving perceptually more similar images.

\section{A. Experimental Study of $m_{p}$}

Since ED, which is a Minkowski-form $\left(l_{p}\right)$ distance where $(p=2)$, has been shown to be the most effective existing dissimilarity metrics in image retrieval, we have chosen this metric as the baseline metric to compare with. In this section, we will compare the image retrieval performance of ED and $m_{p}$. Colour histograms as an important and useful tool for analyzing colour images that are invariant to rotation and translation are used in this work as image features. They carry the statistical information of the chosen colour space and have been used in several image retrieval research works [16-22].

In this study, we use colour histograms obtained from the RGB colour space. Each colour channel is quantized by steps of eight intensity values and this results in 32 bins for each colour channel (i.e. R, G and B), so a total of 96 dimension feature vectors will represent images.

Also, LBP [23] is a very efficient texture operator which labels the pixels of an image by thresholding the neighborhood of each pixel and considers the result as a binary number. Due to its discriminative power and computational simplicity, LBP texture operator has become a popular approach in various applications. We extracted the LBP features of Catlech 101 dataset from neighbourhood of 8 pixels and resulted in a feature vector of size 944 dimensions.

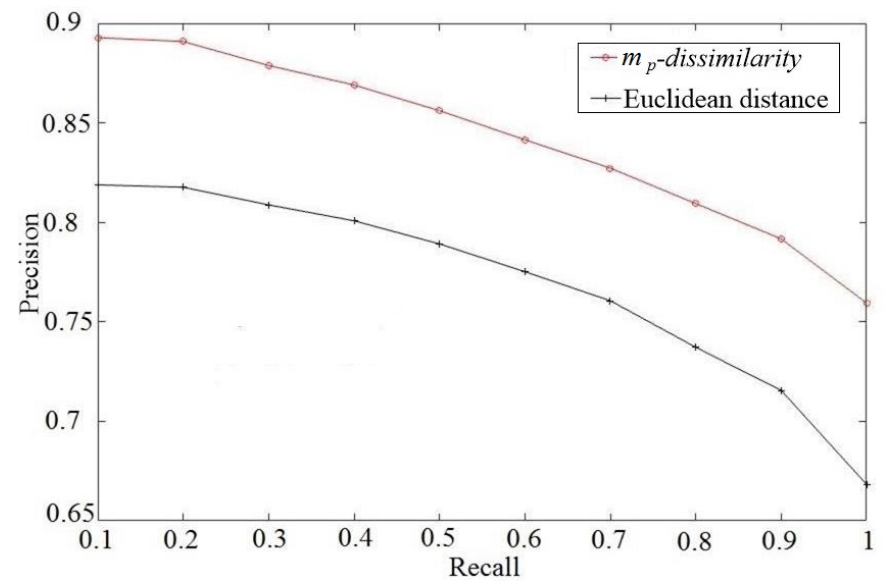

Fig. 2. Precision-recall curves of ED and $m_{p}$ based on the retrieval results for 200 queries from Corel database

\section{B. Overall Performance Comparison}

Image retrieval experiments have been carried out on a version of Corel database [8] that consists of 1000 images from different natural scenes categorized into 10 classes. Images are represented by RGB colour histograms with 32 bins for each colour channel, resulting in a feature vector of 96 dimensions. We randomly selected 200 images from the database as queries. ED and $m_{p}$ have been used as dissimilarity measures to retrieve set of similar images to the query from the database. The performance of image retrieval using each dissimilarity 
measure is evaluated using precision-recall curve. Fig. 2 shows the retrieval results of the 200 query images. It can be seen that $m_{p}$ has produced better retrieval performance than ED.

Also, the experiment has been performed using LBP features and Caltech 101, which has 101 categories of images from different objects and totally $10 \mathrm{k}$ images in the dataset. In this experiment 20 percent of dataset has been used as queries. In this experiment we compared the performance of $m_{p}$ with ED, Cosine and city block distance metrics. Results been shown in Fig 3 shows the better retrieval performance achieved by $m_{p}$.

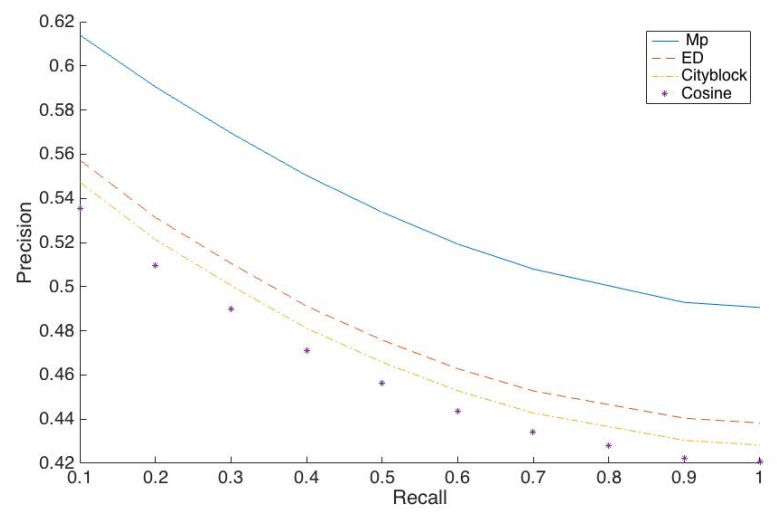

Figure 3. Precision-recall curves of $m_{p}, \mathrm{Ed}$, cosine and cityblock based on the retrieval results for 2000 queries from Caltech 101 database

\section{Visual Examples}

To visually investigate the performance of $m_{p}$ against ED, we present and compare two query images and their top 10 retrieved images as shown in Figs.4-5 from experiment on Corel dataset using color histograms. Visual examples are selection from Corel dataset, which is represented by color histograms to provide better intuition of the results. In each set of images, the top left image is the query and the rest are the 10 highest ranked retrieved images. Generally compared to ED, we can see $m_{p}$ has retrieved sets of images perceptually more similar to the query.

For example in Fig.4.b we can see the influence of dominant colour (the colour of sands in beach query) in the retrieved images. However in Fig.4.a, by using $m_{p}$, we can see the detailed colours (such as red and blue) within the main objects in the query image have played a more prominent role in retrieving perceptually more similar images.

\section{Result analysis}

To provide further insights into retrieving different images using ED and $m_{p}$, we will analyse the colour histograms that are the basis of the calculating ED, as well as the data masses calculated by $m_{p}$

Figs.6-7 show the colour histograms for two query images and their respective top ranked retrieved images using ED and $m_{p}$. As we can see, the difference between the largest values in the colour histogram of the query image (Fig.6.d) and that of the top ranked image of using ED (Fig.6.e) is about 5000. However this difference between the histogram of the query image (Fig.6.d) and that of the top ranked retrieved image using $m_{p}$ (Fig.6.f) is more than 10000. As discussed in Section II A, ED calculates the distance by only considering the values in corresponding dimensions in the colour histograms, and not the distribution of the values in all the colour histograms. As a result, the dominant dimensions will contribute substantially to the total distance calculated, whereas the contribution from the remaining dimensions might be negligible to impact the final retrieval outcome. For example in Fig.6, the colours of the sand (around histogram bins 28, 59 and 90) on the beach of the query image dominate the distance calculated, whereas the detailed colours, such as the skin colours of people (around histogram bins 21, 43 and 74), though perceptually important when comparing similarity between these images, are not dominant enough to impact the final retrieval outcome. As a result, ED has retrieved Fig.6.b as the top rank rather than Fig.6.c. The colour histograms presented in Fig.9 also follow the same trend.

Next, we will analyse the data mass calculated from the two query images and their top ranked retrieved images using ED and $\boldsymbol{m}_{\boldsymbol{p}}$, as shown in Figs.8-9. Data mass is the basis for $\boldsymbol{m}_{\boldsymbol{p}}$ calculation. $\boldsymbol{m}_{\boldsymbol{p}}$ takes into account of data distribution by defining a region and by looking for other images in the same bin that have values falling in this region. $\boldsymbol{m}_{\boldsymbol{p}}$ assigns the maximum dissimilarity (of a dimension) when majority of images having the values falling in the defined region and assigns minimum dissimilarity if less number of images having these values. So, the sparser data mass is considered as the higher similarity.

Figs.8.c and 9.c show the data mass between the feature vectors of each of the two query images and its top ranked retrieved image using $\boldsymbol{m}_{\boldsymbol{p}}$. As we can see, the data mass between the query and top ranked retrieved image using $\boldsymbol{m}_{\boldsymbol{p}}$ is sparser than Figs.8.f and 9.f, which show the data mass of these query images and top ranked retrieved image using ED. Using $\boldsymbol{m}_{\boldsymbol{p}}$, this sparser data mass has resulted in Figs.8.b and 9.b being ranked higher than Figs.7.e and 8.e.

\section{LIMITATION OF $m_{p}$}

In this section we will discuss the limitation that arise when we only rely on data distribution in defining the dissimilarity between two data points. A dissimilarity measure in image retrieval is supposed to retrieve images, which are perceptually similar. As we discussed data distribution has effect on perceptual similarity as considered in $m_{p}$. However the geometric distance between two instances should not be ignored, as that measures the dissimilarity in real world. $m_{p}$ finds two instances similar when the data mass between them is low (they located in a sparse region), in this case we need to find whether they are perceptually similar in real world or not.

Suppose, $m_{p}$ find two instances similar based on low data mass between them but the geometric distance between them is large, they cannot be considered as similar in real world. We expect that when $m_{p}$ finds similarity between two instances 
their geometric distance is small as well. Similarly, we expect when $m_{p}$ find two instances dissimilar due to high data mass between them, their geometric distance be large as well to let us consider them dissimilar in real world.

To provide a better insight we refers to the example provided in section II. In that example we discussed that if we have two red apples among many green apples those two red apples are perceptually more similar than if we place those two red apples among many other red apples. So it shows the effect data distribution in perceptual similarity of two images, which is considered by $m_{p}$. But in another scenario consider we have one red apple among many green apples and we are looking for apples similar to a green apple (query), $m_{p}$ will find the red apple as the most similar one due to low data mass between a green and red apple compare to high data mass between green apples. However the red and green apples are very different and have a great geometric distance. In this case we are not considering any of green apples as the most similar because the data mass is high between any two green apples, however in real world they are similar (they have small geometric distance).

We show this limitation through the following example based on how $m_{p}$ work in each dimension of feature vectors. Table. 1 shows a data distribution in one dimension. Suppose we have a dataset $X=\{x(1), \ldots, x(10)\}$ in $d$-dimensional space, to find the similar data to a query $X$ (Query) $m_{p}$ calculates the data mass in each dimension of feature vectors between query and each of data points in the dataset, then will calculate the total dissimilarity using equation $3 . m_{p}$ will consider the low data mass between two point as lower dissimilarity and high data mass as higher dissimilarity. We will take a closer look at how data mass works in a dimension of feature vectors $x_{i}$. To calculate the data mass, $m_{p}$ defines a region that enclose $\left(x_{i}(\right.$ Query $\left.), x_{i}(j)\right)$ where $1<j<10$ and check how many points in the dataset has the value that fall in this region.

Looking at data mass between query and each data point in the dataset, we will find the lowest data mass ( $x_{i}$ (Query), $\left.x_{i}(10)\right)$ which is 2 . So in this case based on the lowest mass $m_{p}$ will find $x_{i}(10)$ as the closet to the query, however, if we look at their geometric distance $E D\left(x_{i}\right.$ (Query), $\left.x_{i}(10)\right)$, they are very different compare to the rest of data points in the dataset. Hence, $m_{p}$ found the wrong closest match where the data mass between query and a data point was low but they are very different (having large spatial distance). However, there are many other points similar to the query as they have small distance, which are not considered as the closest match because the data mass between query and them was high.

Generally, $m_{p}$ has the limitation to define the dissimilarity when there is small data mass between two points but they are very different (they have large geometric distance). In this case $m_{p}$ will find two different data points as similar while it ignores the similar data points (have small geometric distance) due to high mass between them.

Table 1. Data distribution in one dimension of feature vectors

\begin{tabular}{|c|c|c|}
\hline$X$ & $\ldots$ & $x_{i}$ \\
\hline$x($ Query $)$ & $\ldots$ & 2 \\
\hline$x(1)$ & $\ldots$ & 1 \\
\hline$x(2)$ & $\ldots$ & 1 \\
\hline$x(3)$ & $\ldots$ & 0 \\
\hline$x(4)$ & $\ldots$ & 1 \\
\hline$x(5)$ & $\ldots$ & 1 \\
\hline$x(6)$ & $\ldots$ & 1 \\
\hline$x(7)$ & $\ldots$ & 0 \\
\hline$x(8)$ & $\ldots$ & 0 \\
\hline$x(9)$ & $\ldots$ & 9 \\
\hline$x(10)$ & & \\
\hline
\end{tabular}

\section{HYBRID DATA DEPENDENT DISSIMILARITY (HDDD)}

To this end we have discussed about the advantages of considering data distribution as has been proposed in $m_{p}$ and also the limitation of such a method by completely ignoring the geometric distance in calculation of dissimilarity between two instances. To address the limitation of using $m_{p}$ as a dissimilarity measure, we propose a hybrid data dependent dissimilarity ( $H D D D$ ), which take advantage of data distribution and geometric distance at the same time.

\section{A. Proposed Method for HDDD}

We discussed that $m_{p}$ consider the low data mass between two points as higher similarity (less dissimilarity) and high data mass as less similarity (higher dissimilarity). However, we showed in previous section that if we have a low data mass between two points while the geometric distance between them is large, $m_{p}$ will not find the best closest match. This happens by ignoring the points, which are more similar (having smaller geometric distance), but the data mass between them is high. To address this limitation we need to give a proper weight to the data mass between two points in each dimension.

We need to set a proper weight when data mass between two points is high but they have small geometric distance (they are similar). The weight needs to lower the data mass proportional to the distance between two points. The reason for choosing a weight proportional to the geometric distance between two points and not using a constant weight is as follows. Using $m_{p}$ as a dissimilarity measure, we expect that closest matches to query be similar and meaningful in real world as well. It means the closest matches are desirable to have smaller geometric distance to the query compare to the rest of instances in the dataset. So basically $m_{p}$ should rank its 
closest matches proportional to their distance to the query, means data points with smaller distance should be ranked higher compare to the ones with larger distance. The other reason that the weight should be proportional to distance and cannot be a constant number is as follows. Suppose we have equal data mass between the query point and two other points in the dataset while their geometric distance between are different, we expect to find the point with smaller distance to query as the closest match, however $m_{p}$ based on the equal data mass will find both of them as the closest match. So using a constant weight will result that weighted data mass between them again will be equal which does not help in solving the problem.

We propose to change the equation 3 , to:

$$
\operatorname{HDDD}(x, y)=\left(\frac{1}{d} \sum_{i=1}^{d} a b s\left(W \frac{\left|R_{i}(x, y)\right|}{N}\right)^{p}\right)^{1 / p}
$$

Where $W$ is the weight that lower the data mass when it is high between two points while having small geometric distance, which we set that to $\alpha E D(x, y)$, where $0<\alpha<1$. In this case data mass will be weighted proportional to geometric distance, so when $m_{p}$ search for the closest match it also considers their similarity in real world (geometric distance) along with the data distribution.

\section{B. Experimental study of HDDD}

To evaluate the proposed dissimilarity measure, we will use the two datasets has been used in this work previously, Corel and Caltech 101, and will represent images using LBP features. As we discussed we proposed to use the weight where data mass between query and a point from dataset is high while their spatial distance is small. So to determine the high data mass in each dimension, we consider data mass above the mid point between minimums and maximum of data masses. Also for determining the small spatial distance we consider distances below the mid point between minimum and maximum of distances of query and all data points in each dimension. The $\alpha$ is set to 0.6 as it showed best performance in our experiments. We used ED as the spatial distance as it showed the best performance among other $l_{p}$-norms in Fig 3. The overall retrieval results using $\mathrm{HDDD}, m_{p}$ and $\mathrm{ED}$ for Corel and Caltech 101 datasets are shown in Figs 10-11.

As it can be seen the retrieval results has been improved using HDDD over $m_{p}$ and ED.

\section{Visual Examples}

In this section we present visual examples to give a better insight about the performance of HDDD against $m_{p}$, we present and compare two query images and their top 10 retrieved images as shown in Figs.12-13 images are selected from Corel dataset represented by LBP features. Using visual examples we can see how our proposed dissimilarity measure could address the discussed limitation of $m_{p}$. In each set of images, the top left image is the query and the rest are the 10 highest ranked retrieved images. Generally compared to $m_{p}$, $H D D D$ has retrieved sets of images perceptually more similar to the query.

For example in Fig 12.a, retrieved images in ranks 1-2 are not form the same class with query and this has been improved in Fig 12.b using $H D D D$. Also in Fig 13.a, retrieved images in ranks 4-5 belong to a different class with query while in Fig 13.b all the retrieved images are from the same class with query.

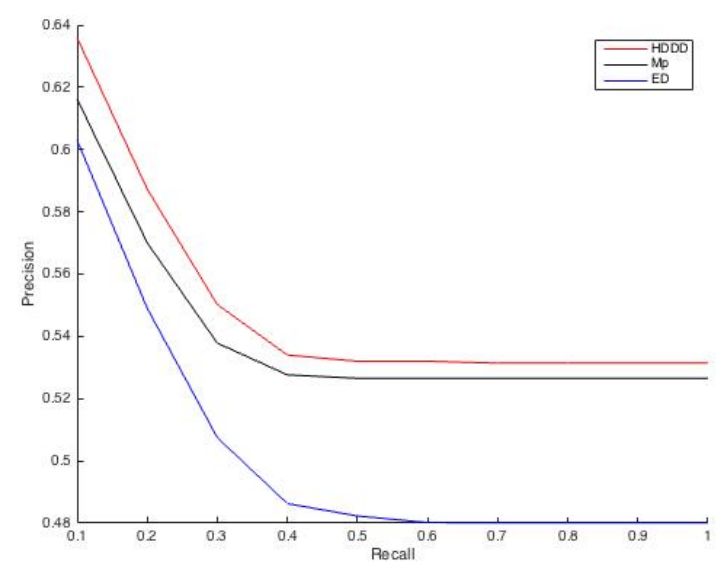

Figure 10. Precision-recall curves of $H D D D, m_{p}$ and Ed based on the retrieval results from Corel.

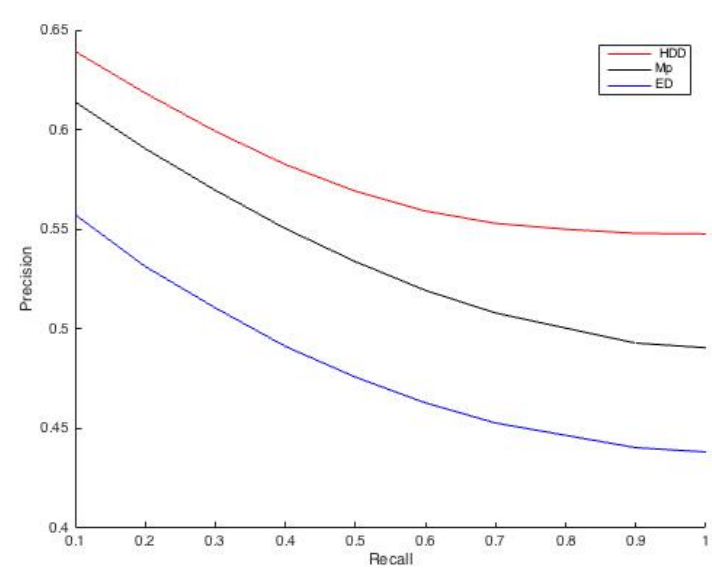

Figure 11. Precision-recall curves of $H D D D, m_{p}$ and Ed based on the retrieval results from Caltech 101 .

\section{Result Analysis}

In this section we show the limitation of $m_{p}$ in completely ignoring the spatial distance between two images and how $H D D D$ could improve it. $m_{p}$ is calculated based on the average data mass in all dimensions between query and each dataset image, so smaller data mass in each dimension will result in smaller $m_{p}$. In Fig 12.a $m_{p}$, ranked retrieved images based on data mass between query and each image in the dataset, the smaller data mass made the elephant $\left(m_{p}=6.43\right)$ and the other irrelevant image from food class $\left(m_{p}=\right.$ 6.53) come up in the first and second rank compare to the 
relevant image in the third rank where $\left(m_{p}=6.59\right)$. However, the Euclidean distance between query, elephant $(E D=3.8)$ and food dish $(E D=2.9)$ are larger than distance between query and third rank which is relevant image $(E D=1.01)$. So ignoring the distance and only relying on data mass caused that smaller data mass retrieved images, which are very different with query in highest ranks.

The same scenario is in Fig 13.a where $m_{p}$ retrieved images based on lower data mass in high ranks while they are different and having larger distance to the query compare to the relevant images that are ranked lower due to the higher data mass (while having relatively smaller distance to the query). Data masses between query, rank 4 and 5 are $\left(m_{p}=6.72,7.03\right)$ which are lower than data mass between query and rank $6\left(m_{p}=7.8\right)$ however ED for the formers are 2.6, 2.3 Which is much larger than ED for latter,1.3.

As we showed relying only on data mass between two instances, may result in following situation: $m_{p}$ ranks instances with lower data mass but different with query (large distance) higher than instances similar to the query (small distance) which has higher data mass. In $H D D D$, we used the ED as the weight in each dimension where data mass is high between query and dataset image but the distance is small. This improved the result by ranking those images higher than the ones with small data mass but large distance. The results in Figs 12.13.b show this effect visually.

\section{CONCLUSIONS}

In this work, we studied $m_{p}$ strengths and limitations as a data dependent dissimilarity measure for image retrieval. Our experimental results show that $m_{p}$ outperforms ED, Cosine and cityblock distance in retrieving perceptually more similar images. We also showed the limitation of $m_{p}$ by completely ignoring the spatial distance and only relying on data distribution. This could result in retrieving irrelevant images in high ranks, which has large distance to the query by only considering low data mass between them. We proposed a new hybrid data dependent dissimilarity measure by considering both data distribution and spatial distance. The proposed dissimilarity measure could perform better than $m_{p}$ and yield perceptually more similar retrieved images.

\section{REFERENCES}

1. Zhang, D., M.M. Islam, and G. Lu, A review on automatic image annotation techniques. Pattern Recognition, 2012. 45(1): p. 346-362.

2. Zhang, D. and G. Lu, Review of shape representation and description techniques. Pattern Recognition, 2004. 37(1): p. $1-19$.

3. Aryal, S., et al. Mp-Dissimilarity: A Data Dependent Dissimilarity Measure. in Data Mining (ICDM), 2014 IEEE International Conference on. IEEE.
4. Rogers, P.L., Encyclopedia of distance learning. 2009: IGI Global.

5. Krumhansl, C.L., Concerning the applicability of geometric models to similarity data: The interrelationship between similarity and spatial density. 1978.

6. Zhang, D. and G. Lu. Evaluation of similarity measurement for image retrieval. in Neural Networks and Signal Processing, 2003. Proceedings of the 2003 International Conference on. 2003. IEEE.

7. Malik, F. and B. Baharudin, Analysis of distance metrics in content-based image retrieval using statistical quantized histogram texture features in the DCT domain. Journal of King Saud University-Computer and Information Sciences, 2013. 25(2): p. 207-218.

8. $\quad$ Wang, J.Z., Wang dataset.

9. Bosch, A., A. Zisserman, and X. Muñoz, Scene classification via pLSA, in Computer Vision-ECCV 2006. 2006, Springer. p. 517-530.

10. Jain, A.K. and A. Vailaya, Image retrieval using color and shape. Pattern Recognition, 1996. 29(8): p. 1233-1244.

11. Khotanzad, A. and Y.H. Hong, Invariant image recognition by Zernike moments. Pattern Analysis and Machine Intelligence, IEEE Transactions on, 1990. 12(5): p. 489497.

12. Kim, H.-K., et al. A modified Zernike moment shape descriptor invariant to translation, rotation and scale for similarity-based image retrieval. in Multimedia and Expo, 2000. ICME 2000. 2000 IEEE International Conference on. IEEE.

13. Singh, C., Local and global features based image retrieval system using orthogonal radial moments. Optics and Lasers in Engineering, 2012. 50(5): p. 655-667.

14. Kruskal, J.B., Nonmetric multidimensional scaling: a numerical method. Psychometrika, 1964. 29(2): p. 115-129.

15. Aryal, S., et al., Data-dependent dissimilarity measure: an effective alternative to geometric distance measures. Knowledge and Information Systems, 2017: p. 1-28.

16. Saad, M.H., et al., Image Retrieval Based on Integration Between $\mathrm{YCbCr}$ Color Histogram and Texture Feature. International Journal of Computer Theory and Engineering, 2011. 3(5): p. 701

17. Vailaya, A., et al., Image classification for content-based indexing. Image Processing, IEEE Transactions on, 2001. 10(1): p. 117-130.

18. Fan, J., et al. Automatic image annotation by using concept-sensitive salient objects for image content representation. in Proceedings of the 27 th annual international ACM SIGIR conference on Research and development in information retrieval. ACM.

19. Feng, S.L., R. Manmatha, and V. Lavrenko. Multiple bernoulli relevance models for image and video annotation. in Computer Vision and Pattern Recognition, 2004. CVPR 2004. Proceedings of the 2004 IEEE Computer Society Conference on. IEEE.

20. Yang, C., M. Dong, and F. Fotouhi. Image content annotation using Bayesian framework and complement components analysis. in Image Processing, 2005. ICIP 2005. IEEE International Conference on. IEEE.

21. Goh, K.-S., E.Y. Chang, and B. Li, Using one-class and two-class SVMs for multiclass image annotation. Knowledge and Data Engineering, IEEE Transactions on, 2005. 17(10): p. 1333-1346.

22. Jeong, S., C.S. Won, and R.M. Gray, Image retrieval using color histograms generated by Gauss mixture vector 
quantization. Computer Vision and Image Understanding, 2004. 94(1): p. 44-66.

23. Ojala, T., M. Pietikainen, and T. Maenpaa, Multiresolution gray-scale and rotation invariant texture classification with local binary patterns. IEEE Transactions on pattern analysis and machine intelligence, 2002. 24(7): p. 971-987.

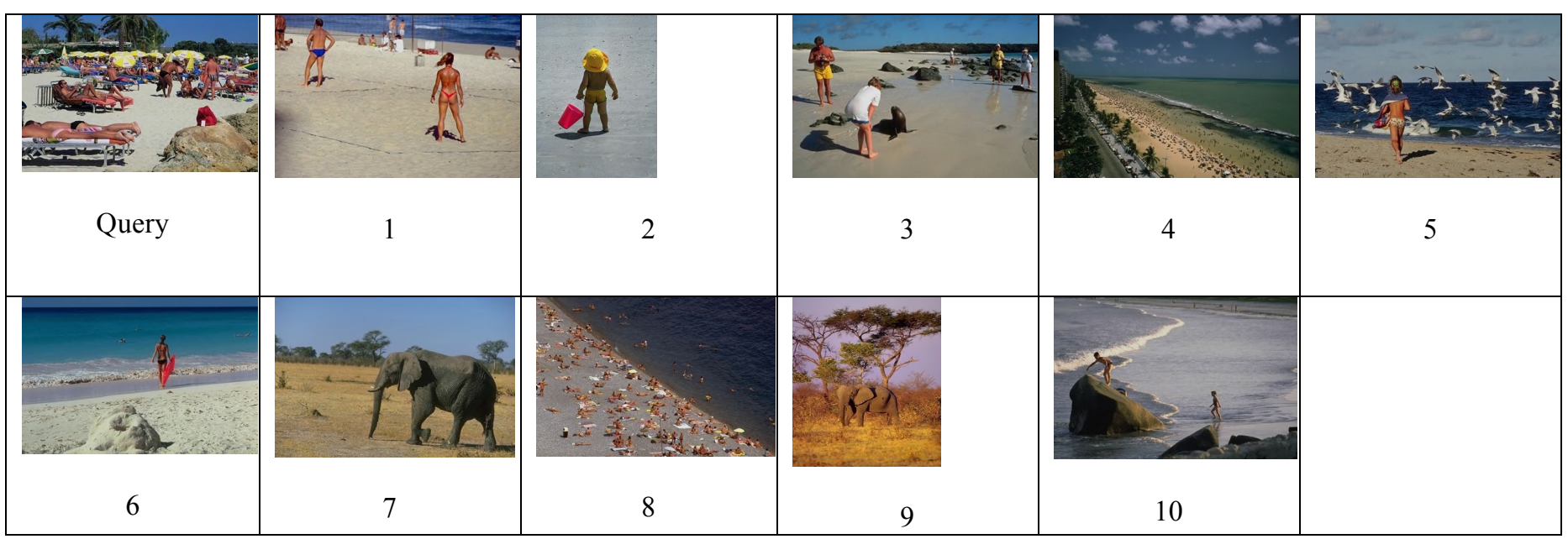

(a) using $\boldsymbol{m}_{p}$

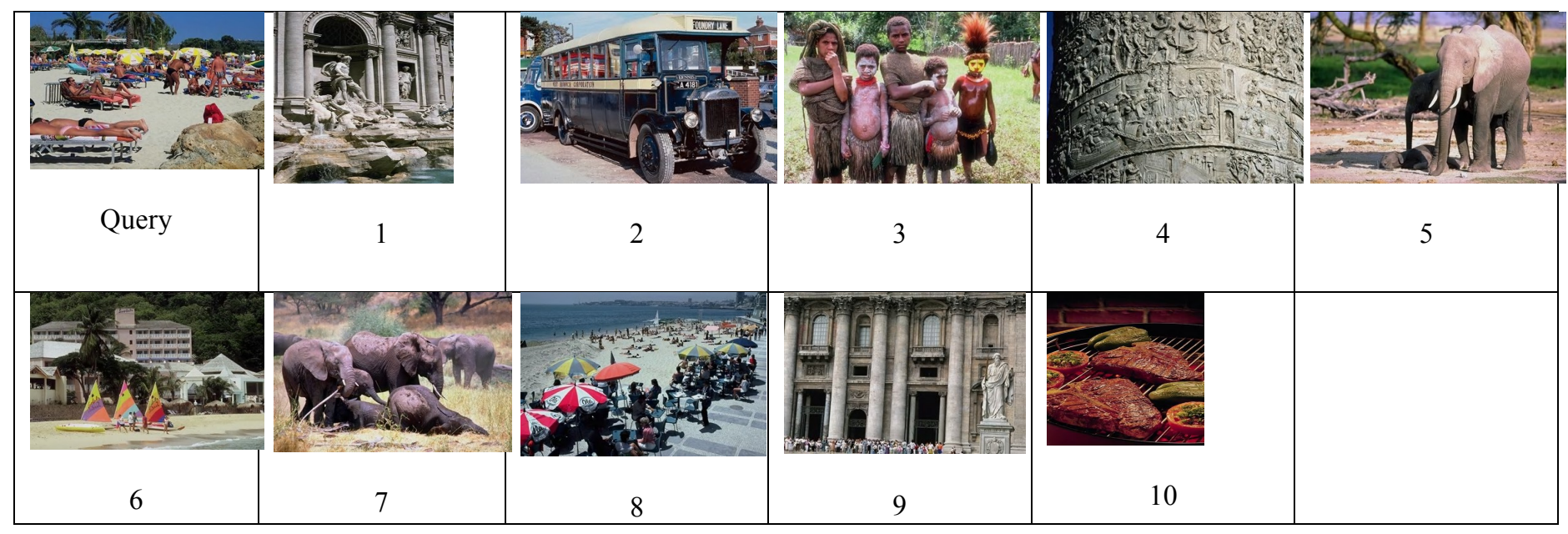

(b) using ED

Figure 4. Top 10 retrieval for Query 1. 


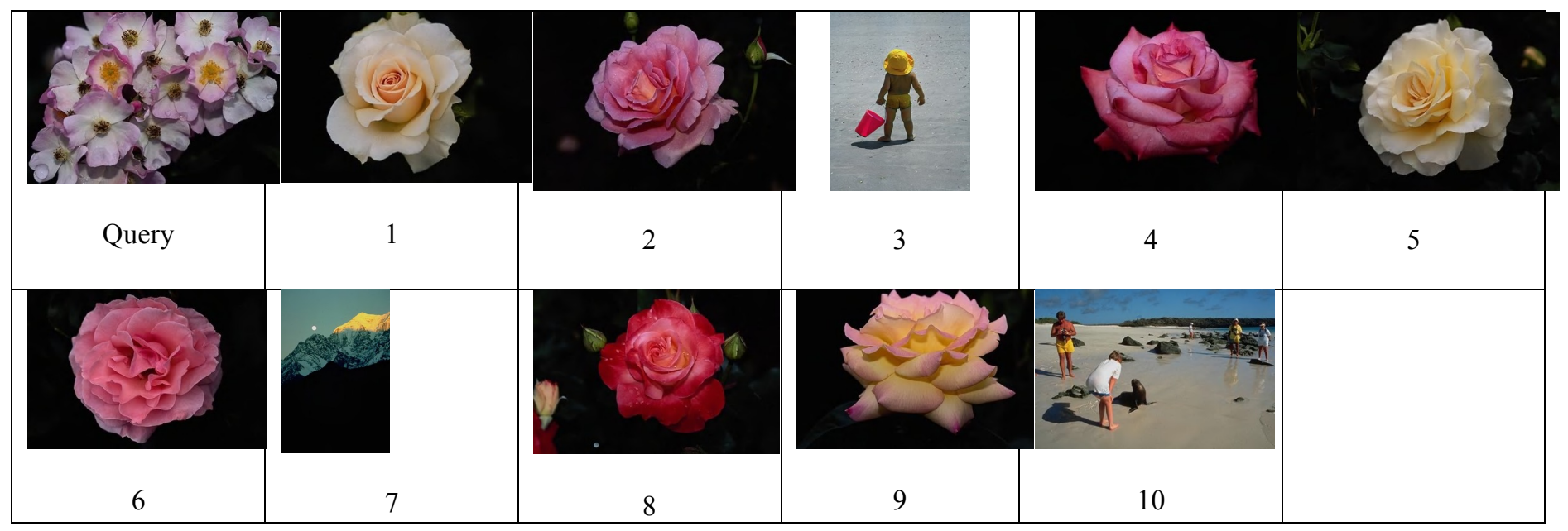

(a) using $\boldsymbol{m}_{p}$

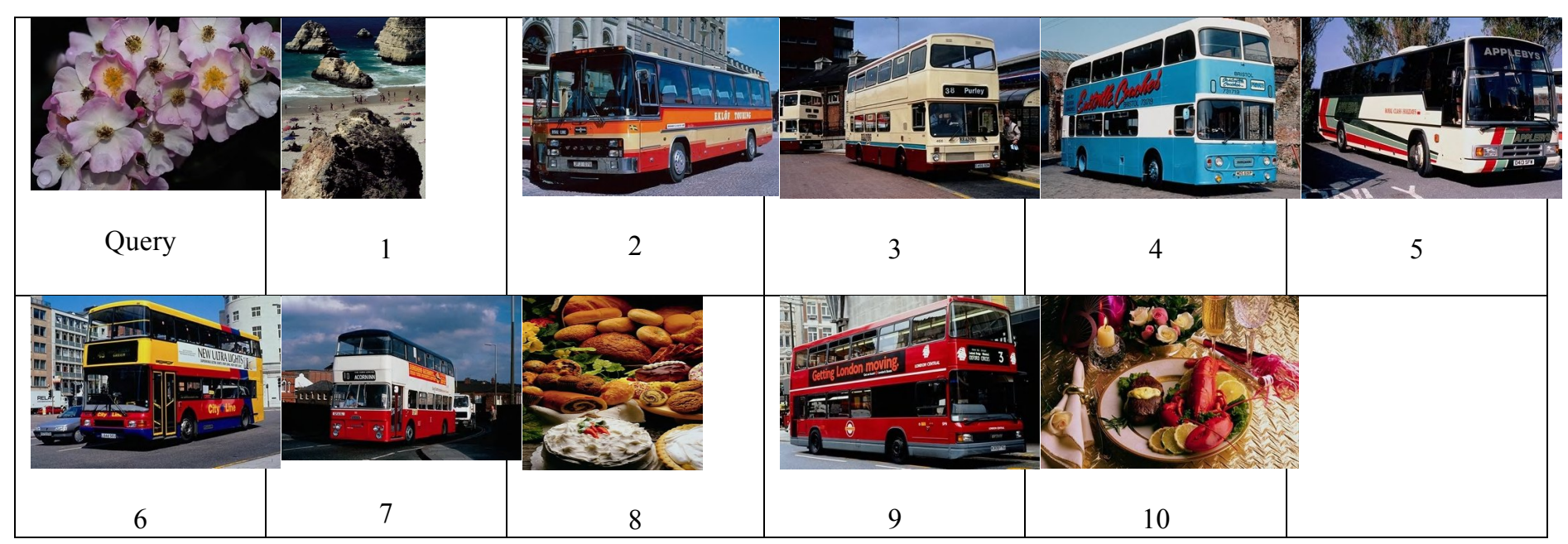

(b) using ED

Figure 5. Top 10 retrieval for Query 2. 


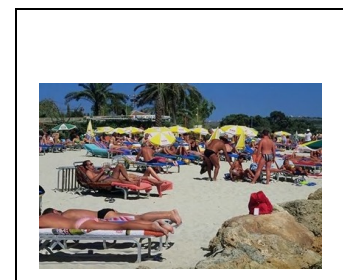

(a) Query

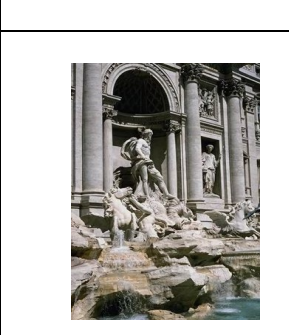

(b) Top rank retrieved
images form ED

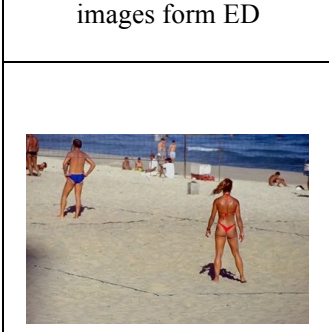

(c) Top rank retrieved images form $\boldsymbol{m}_{p}$

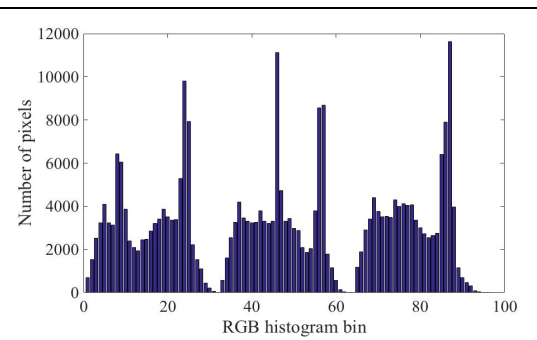

(d) Colour histograms of query

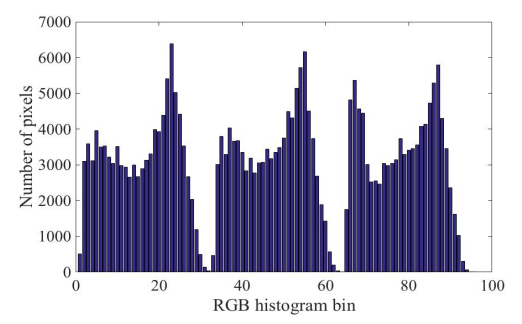

(e) Colour histograms of top rank retrieved images form ED

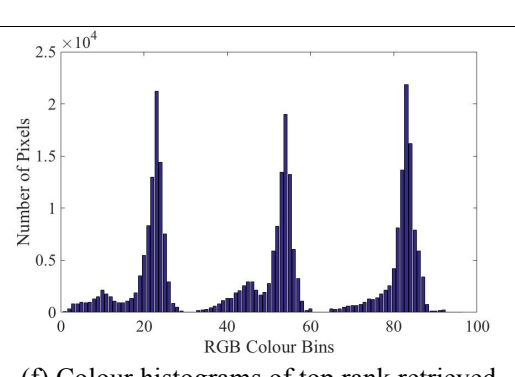

(f) Colour histograms of top rank retrieved images form $\boldsymbol{m}_{p}$

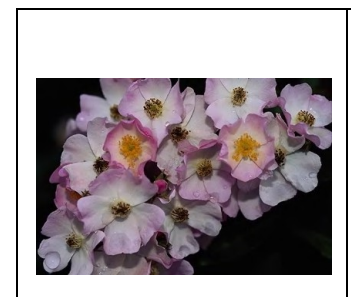

(a) Query
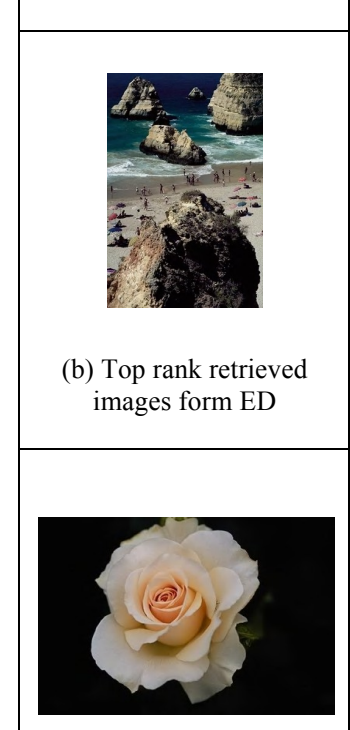

(c) Top rank retrieved images form $\boldsymbol{m}_{p}$

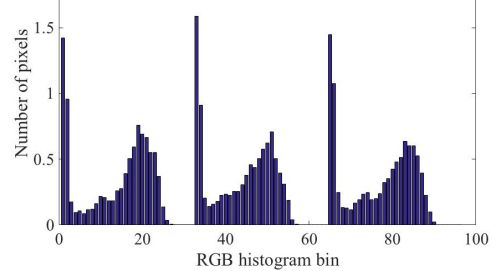

(d) Colour histograms of query

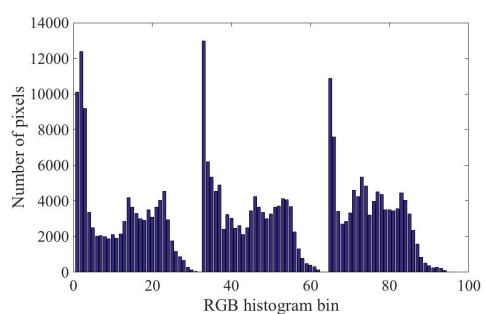

(e) Colour histograms of top rank retrieved images form ED

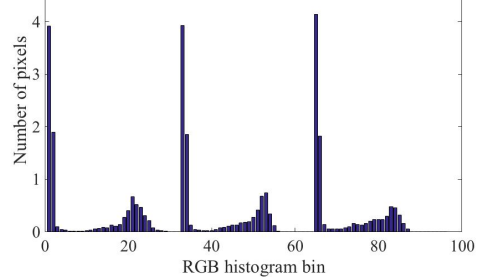

(f) Colour histograms of top rank retrieved images form $\boldsymbol{m}_{p}$

Figure 6. Colour histogram comparison of query 1

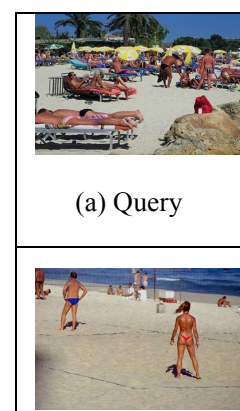

(b) Top rank retrieved images form $\boldsymbol{m}_{p}$

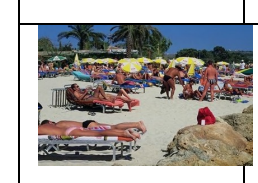

(d) Query

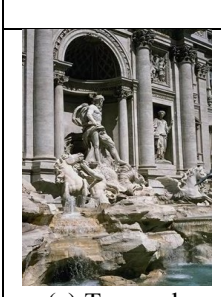

(e) Top rank retrieved images form ED

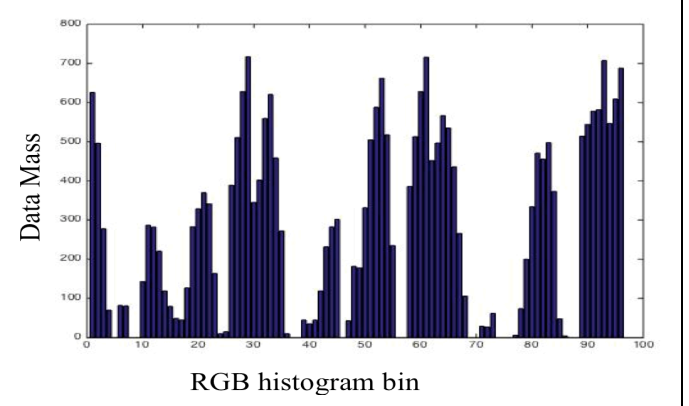

(c) Data mass between (a) and (b)

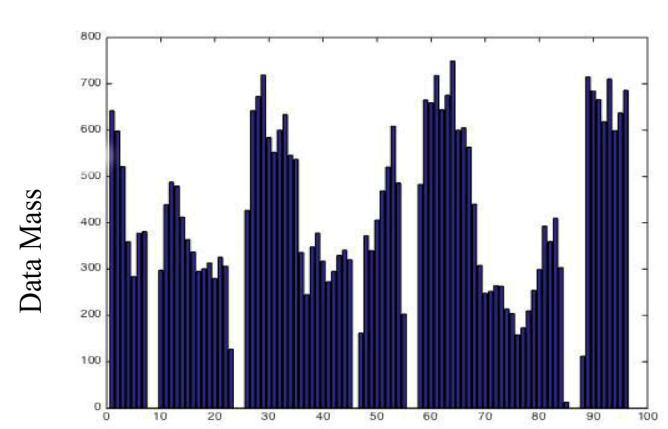

RGB histogram bin

(f) Data mass between (d) and (e)

Figure 7. Colour histogram comparison of query 2

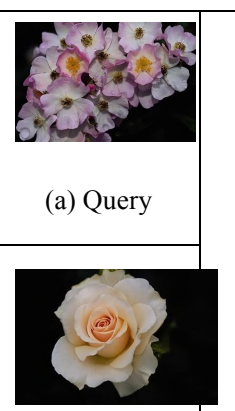

(b) Top rank

retrieved images form

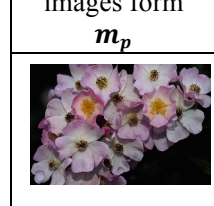

(d) Query

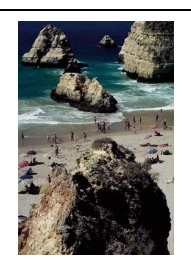

(e) Top rank

retrieved images form

ED

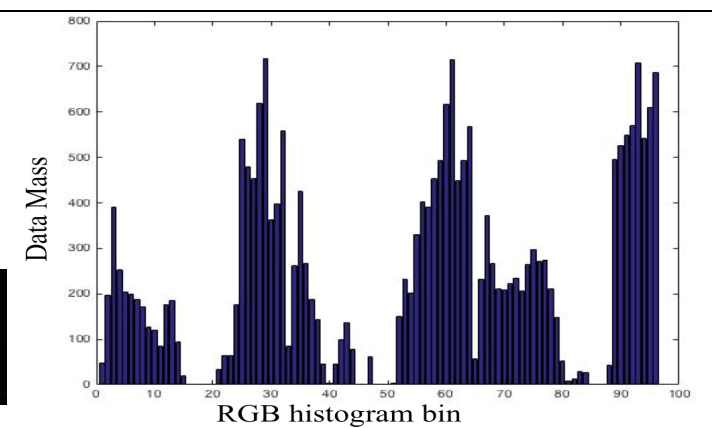

(c) Data mass between (a) and (b)

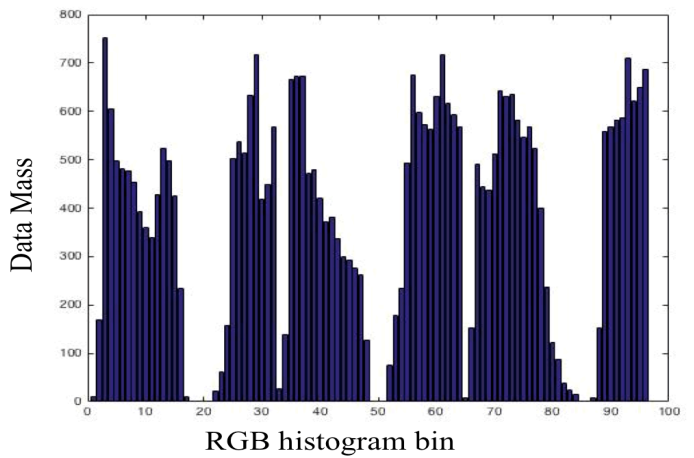

(f) Data mass between (d) and (e) 


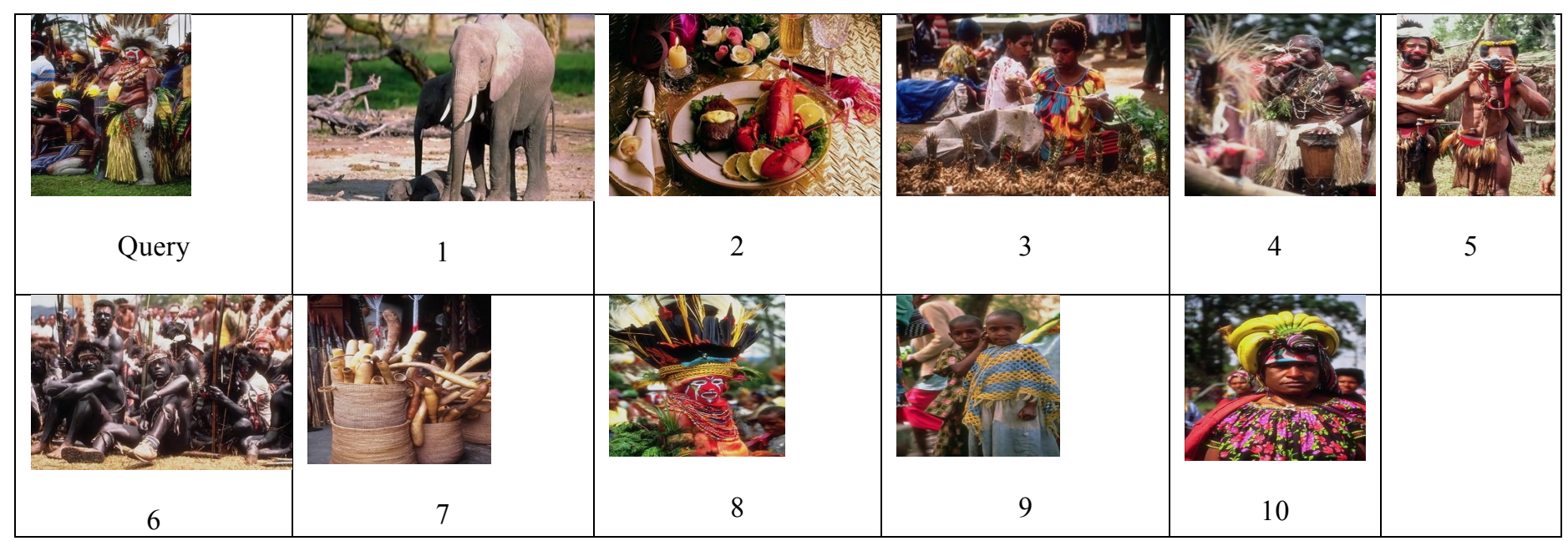

(a) using $\boldsymbol{m}_{\boldsymbol{p}}$

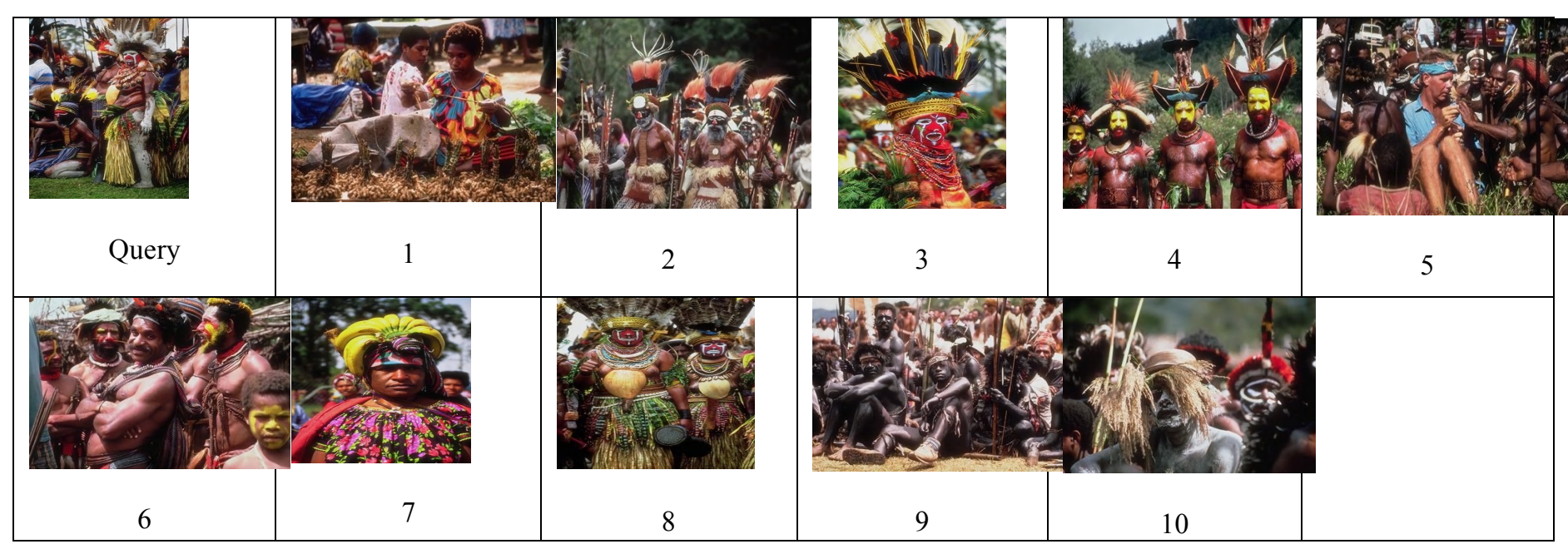

(b) using HDDD

Figure 12 . Top 10 retrieval for Query 1.

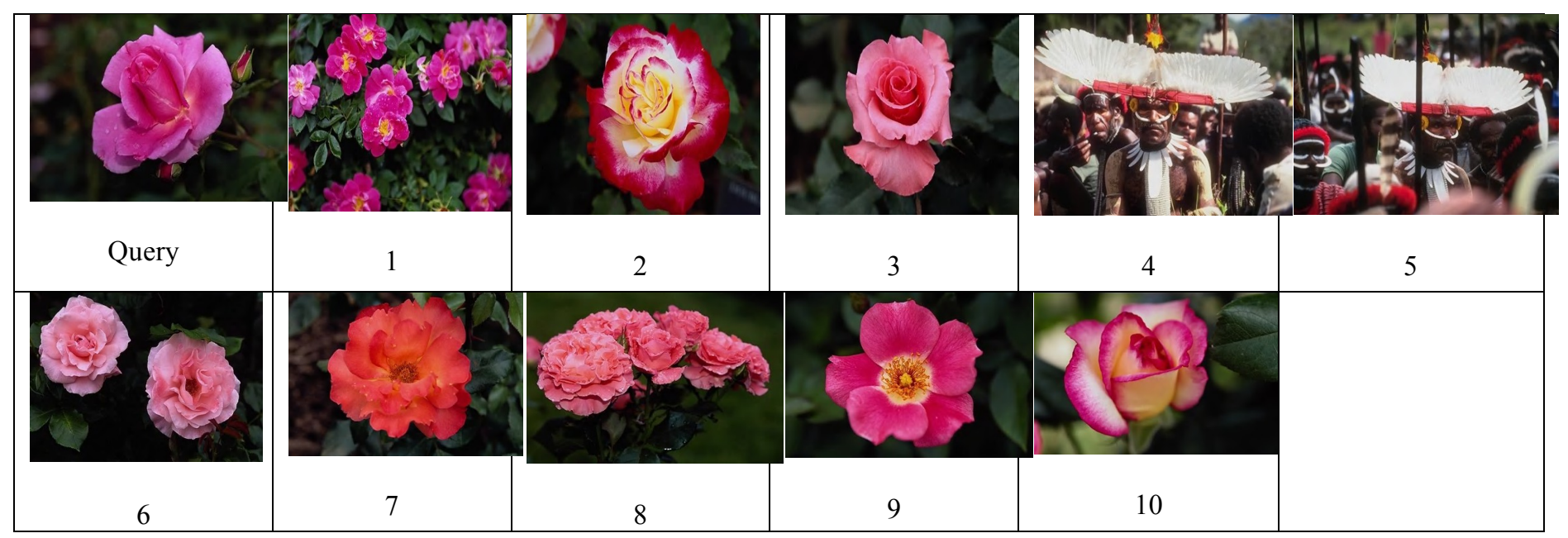

(a) using $\boldsymbol{m}_{\boldsymbol{p}}$ 


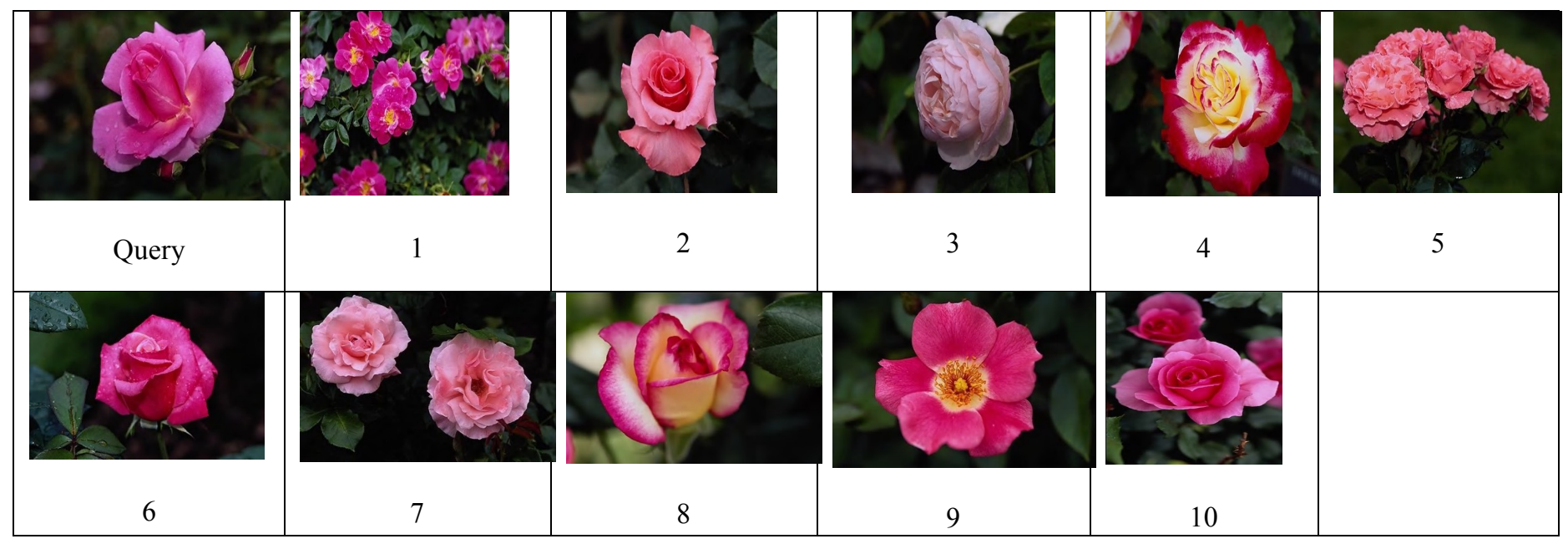

(b) using HDDD

Figure 13. Top 10 retrieval for Query 2 